\title{
MINIMALITY AND UNIQUE ERGODICITY FOR ADIC TRANSFORMATIONS
}

\author{
SEBASTIEN FERENCZI, ALBERT M. FISHER AND MARINA TALET
}

\begin{abstract}
We study the relationship between minimality and unique ergodicity for adic transformations. We show that three is the smallest alphabet size for a unimodular "adic counterexample", an adic transformation which is minimal but not uniquely ergodic. We construct a specific family of counterexamples built from $(3 \times 3)$ nonnegative integer matrix sequences, while showing that no such $(2 \times$ 2 ) sequence is possible. We also consider $(2 \times 2)$ counterexamples without the unimodular restriction, describing two families of such maps.

Though primitivity of the matrix sequence associated to the transformation implies minimality, the converse is false, as shown by a further example: an adic transformation with $(2 \times 2)$ stationary nonprimitive matrix, which is both minimal and uniquely ergodic.
\end{abstract}

Research of the authors was partly supported by: the CNPQ, the Acordo FrancoBrasil, FAPESP, and the CNRS.

\section{INTRODUCTION}

Adic transformations, as defined by Vershik [Ver89], offer a far-reaching generalization of the classical odometer (or adding machine) transformation of Kakutani and von Neumann. Indeed, on a measure-theoretic level they are completely general: as Vershik showed [Ver81], [LV92], any invertible measure-preserving transformation of a Lebesgue space has an adic model. Moreover this can be constructed so as to be strictly ergodic: to be both minimal (every orbit is dense) and uniquely ergodic (that there is a unique invariant probability measure), thus giving a new proof of the Jewett-Krieger theorem that every ergodic transformation has a strictly ergodic model [Jew70], [Kri70].

The focus of this paper and its companion paper [Fis08] is the relationship between the properties of minimality and unique ergodicity for transformations of a topological space, making use of the framework of adic transformations. In this study Vershik's general theorem will be of no use; as it is purely measure-theoretic in nature, it erases other aspects of the map being studied. Instead we find ourselves involved with a quite different aspect of the theory of adic transformations: that of finding adic models which are naturally adapted to the geometry or topology of a given transformation. In a variety of cases, one can indeed find such adic models; examples we shall encounter below include cutting and stacking constructions, substitution dynamical systems, and interval exchange transformations.

Date: December 28, 2008 (to appear, Journal d'Analyse Mathematique, 2009).

Key words and phrases. Unique ergodicity, adic transformation. 
The first part of the paper concerns the construction of adic transformations which though minimal are not uniquely ergodic. There is a long and interesting history of maps with this feature, including the torus skew product of Furstenberg [Fur61] and the Keynes-Newton and Keane interval exchange transformations [KN76], [Kea77]. Our approach will make use of criteria for minimality and unique ergodicity from [Fis08] and explained below.

In the final part we study a specific adic transformation inspired by a substitution dynamical system of [Fer95] which in turn is closely related to Chacon's famous map of the interval, constructed by cutting and stacking in [Cha69].

But first we recall the basic framework for Vershik's maps. Letting $\left(\mathcal{A}_{i}\right)_{i \geq 0}$ be a sequence of finite alphabets with with $\# \mathcal{A}_{i}=l_{i}$ and $\left(M_{i}\right)_{i \geq 0}$ a sequence of $\left(l_{i} \times l_{i+1}\right)$ nonnegative integer matrices, we construct a Bratteli diagram with vertices $\mathcal{A}_{i}$ at level $i$ and with $\left(M_{i}\right)_{k j}$ directed edges from symbol $k \in \mathcal{A}_{i}$ to $j \in A_{i+1}$; we call $\mathcal{E}_{i}$ the set of edges from level $i$ to level $(i+1)$. We denote by $\Sigma_{(M)}^{+, 0}$ the set of all allowed infinite edge paths $\underline{e}=\left(. e_{0} e_{1} \ldots\right)$ in this diagram; this is a Markov compactum in Vershik's terminology. An incoming edge order (or just order) $\mathcal{O}$ on the Bratteli diagram is a total order on the collection of all edges which enter a given symbol at some level $k$. We define $W^{s}(\underline{e})=\left\{\underline{\tilde{e}} \in \Sigma_{(M)}^{+, 0}: \exists k \geq 0\right.$ with $e_{i}=\tilde{e}_{i}$ for all $\left.i \geq k\right\}$; these collections partition the space.

Vershik's transformation will be defined as a map on the Markov compactum; the orbit of a point $\underline{e}$ will be its stable equivalence class or stable manifold $W^{s}(\underline{e})$.

Beginning with the edge order $\mathcal{O}$, we next place a total order on each $W^{s}(\cdot)$ as follows: given $\underline{\tilde{e}} \in W^{s}(\underline{e})$, let $k$ be the least integer such that $e_{i}=\tilde{e}_{i}$ for all $i \geq k$, and define $\underline{e}<\underline{\tilde{e}}$ iff $e_{k-1}<\tilde{e}_{k-1}$ in the edge order $\mathcal{O}$ at that level.

This is $\mathcal{O}$-lexicographic order; the map $T_{\mathcal{O}}$ is defined to send a path $\underline{e}$ to its sucessor. Writing $\mathcal{N S}$ for the collection of paths with no successor and $\mathcal{N} \mathcal{P}$ for those with no predecessor, then $T_{\mathcal{O}}$ is a bijection from $\Sigma_{(M)}^{+} \backslash \mathcal{N S}$ to $\Sigma_{(M)}^{+} \backslash \mathcal{N} \mathcal{P}$. Defining $\mathcal{N}$ to be the forward and backward images under iteration of these sets, then $T_{\mathcal{O}}$ is a bijection on $\Sigma_{(M)}^{+} \backslash \mathcal{N}$. The number of points in $\mathcal{N} \mathcal{P}$ and $\mathcal{N S}$ are bounded above by $\lim \sup l_{i}$ if that is finite, and in any case is countably infinite, as is $\mathcal{N}$.

Although the space $\Sigma_{(M)}^{+, 0} \backslash \mathcal{N}$ on which the adic transformation is defined for all time is noncompact, it still makes sense to speak of minimality and unique ergodicity in this context, with the usual definitions.

One can also define related dynamics on the whole space $\Sigma_{(M)}^{+, 0}$, and then compare these properties for the different actions. There are two basic ways of doing this: by considering extensions of the map to the whole space, or by replacing the action of the map by that of a related group of homeomorphisms defined on all of $\Sigma_{(M)}^{+, 0}$, the group $\mathcal{F C}$ of finite coordinate changes. Since $\mathcal{N}$ is countable, it follows that essential minimality and essential unique ergodicity correspond for all three actions (the adic transformation on $\Sigma_{(M)}^{+, 0} \backslash \mathcal{N}$, any extension to $\Sigma_{(M)}^{+, 0}$, or the group $\mathcal{F C}$ ) by which we mean respectively minimal off of a countable set, and that there exists a unique invariant nonatomic probability measure; with the assumption of primitivity (see below for the definition) the nonatomic restriction can be removed. So in particular these properties are independent of the particular order $\mathcal{O}$ placed on the diagram, 
and are determined solely by the matrix sequence $\left(M_{i}\right)_{i \geq 0}$. See Proposition 2.10 of [Fis08].

Regarding extensions of the map, the most natural choice will be to take a continuous extension; however we note that even for simple examples this may not be possible, see the discussion below of circle rotations and of the Chacon adic map.

Though we have termed $W^{s}(\underline{e})$ the "stable manifold" of a point, a priori this makes no sense, as the nonstationary combinatorics means there is no actual shift dynamics: a left shift map $\sigma$ should send an edge path $\underline{e}=\left(e_{0}, e_{1}, \ldots\right)$ to $\sigma(\underline{e})=\left(e_{1}, e_{2}, \ldots\right)$; however $\sigma(\underline{e})$ is not an element of the same the Markov compactum $\Sigma_{(M)}^{+, 0}$. So what we do is to simply enlarge the space as follows: writing $\sigma(M)_{i \geq 0}=\left(M_{i+1}\right)_{i \geq 0}$ for the shifted sequence of matrices, we set $\Sigma_{(M)}^{+, k} \equiv \Sigma_{\sigma^{k}(M)}^{+, 0}$. We then form the disjoint union $\Sigma_{(M)}^{+} \equiv \coprod_{k=0}^{\infty} \Sigma_{(M)}^{+, k}$, the nonstationary subshift of finite type defined by the matrix sequence; $\Sigma_{(M)}^{+, k}$ is called the $k^{\text {th }}$ component of $\Sigma_{(M)}^{+}$. This has been constructed so the map $\sigma$ now takes the $k^{\text {th }}$ component of the nsft to the $(k+1)^{\text {st }}$ component. With this definition, and with respect to a natural metric which makes each component a Cantor set, $W^{s}(\underline{e})$ is indeed the "stable manifold" of that point with respect to this mapping family or nonstationary dynamical system, see [AF05]; the stable manifolds subdivide each component into equivalence classes, and the map $T_{\mathcal{O}}$ extends to the entire $n s f t$, preserving each such equivalence class.

In the case when the $M_{i}$ are $0-1$ matrices (so equivalently the diagram has no multiple edges) we can replace the edge shift space defined above by a vertex shift space, as an edge path $\left(e_{0}, e_{1}, \ldots\right)$ determines a unique vertex path $\left(x_{0}, x_{1} \ldots\right)$ where $x_{i}$ is the "tail" of the arrow $e_{i}$. The simplest situation is a stationary adic transformation, where the alphabets, matrices and orders are constant. Thus $M$ is a square matrix, and the $n s f t \Sigma_{(M)}^{+}$factors onto its zeroth component $\Sigma_{(M)}^{+, 0}$ which in turn is canonically identified with the subshift of finite type $(s f t) \Sigma_{M}^{+}$. Note that the Bratteli diagram then factors onto (and can be replaced by) the usual graph of an sft, and that the sft comes in either an edge or vertex presentation (see e.g. [LM95]) depending as to whether or not this graph has multiple edges.

There is an intimate relation between the adic transformation framework and that of substitution dynamical systems, which we now describe. In particular this connection led us to the final example studied below, the Chacon adic transformation.

Given an ordered Bratteli diagram, we note that the information furnished by the matrix sequence $\left(M_{i}\right)_{i \geq 0}$ together with the order $\mathcal{O}$ can instead be conveniently specified by a sequence $\left(\rho_{i}\right)_{i \geq 0}$ of substitutions $\rho_{i}: \mathcal{A}_{i+1} \rightarrow \mathcal{A}_{i}^{*}$, where $\mathcal{A}_{i}^{*}$ denotes the finite words on that alphabet: if $\rho_{k}(j)=a_{0} a_{1} \ldots a_{n}$, then there are $n$ edges entering symbol $j$, ordered as their tail symbols $a_{i}$ appear from left to right. Here we should visualize the substitution sequence as acting from right to left in the Bratteli diagram, the opposite of the "future" direction indicated by the directed edges. By definition, the matrix of the substitution $\rho_{k}$ is then the $\left(l_{k} \times l_{k+1}\right)$ matrix $M_{k}$ with $\left(M_{k}\right)_{i j}$ the number of occurences of the letter $i$ in $\rho_{k}(j)$; note that this gives exactly the matrix sequence used to define the Bratteli diagram.

For the case of a single substitution $\rho: \mathcal{A} \rightarrow \mathcal{A}^{*}$, we recall how one defines from this a substitution dynamical system; then we describe the canonical connection between 

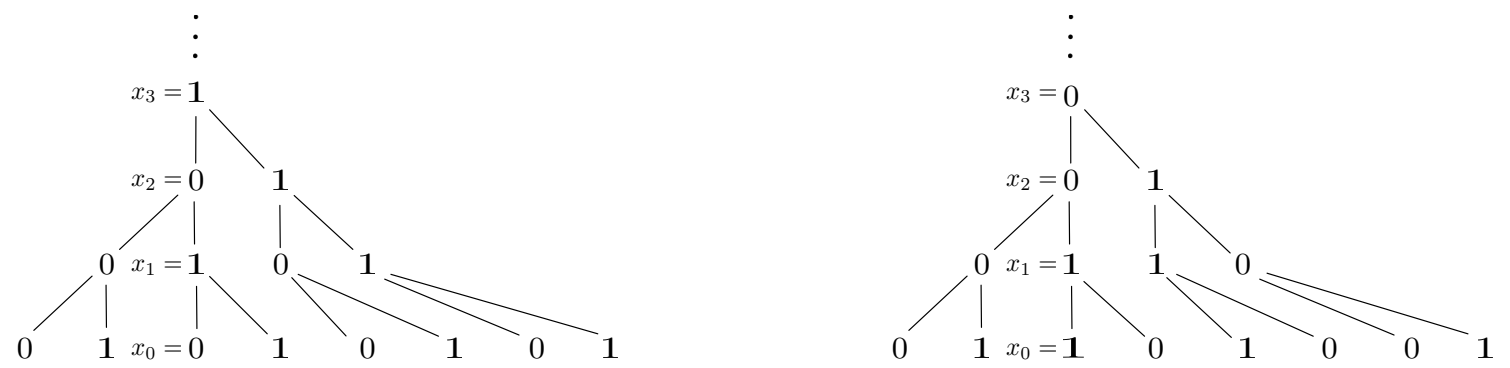

FiguRE 1. Stable trees for the odometer and Morse adic transformations.

stationary adic transformations and substitution dynamical systems, first noted by Livshits [Liv87], [Liv88].

First we extend this map to $\rho: \mathcal{A}^{*} \rightarrow \mathcal{A}^{*}$ by concatenation; then choosing some symbol $a \in \mathcal{A}$ and iterating, the collection of finite words $\rho^{n}(j)$ will converge either to a fixed point (iff $\rho(a)$ begins with $a$ ) or to a periodic orbit; for $\underline{a}=\left(. a_{0} a_{1} \ldots\right.$ ) one of these one-sided infinite words in $\prod_{i=0}^{\infty} \mathcal{A}$, we let $\Omega_{\underline{a}}$ denote the orbit closure in the natural sense of $\underline{a}$ for the left shift map $S$ on the biinfinite space $\Pi_{-\infty 0}^{\infty} \mathcal{A}$. Note that if the matrix $M$ of $\rho$ is primitive (i.e. there exists $n>0$ such that all the entries of $M$ are strictly positive), then $\Omega_{\underline{a}}$ does not depend on the choice of the letter $a$.

Two simple examples which already illustrate the relationship noted by Livshits as well as various other general aspects of the theory are the odometer transformation and the Morse adic transformation. These are both stationary adic transformations, with alphabet $\mathcal{A}=\{0,1\}$ and matrix $M=\left[\begin{array}{ll}1 & 1 \\ 1 & 1\end{array}\right]$, so $\Sigma_{M}^{+}$is a full one-sided Bernoulli shift space (here we take the vertex representation). For the adding machine we order the edges entering the symbol 0 by $00<10$, and those entering 1 by $01<11$, i.e. in both cases just by the natural order on the alphabet. For the Morse transformation, we have edges ordered by: $00<10$, but $11<01$. The associated substitutions are: for the odometer $\rho(j)=01$ for all $j$, for the Morse adic $\rho(0)=01, \rho(1)=10$.

To describe the relationship to substitution dynamical systems we first recall a geometric picture of the adic dynamics, given by the stable tree model [Fis08]; we describe this for a $0-1$ matrix sequence $\left(M_{i}\right)_{i \geq 0}$. Choosing $\left(. x_{0} x_{1} \ldots\right) \in \Sigma_{(M)}^{+}$(in the vertex representation, so $x_{i} \in \mathcal{A}_{i}$ ) we draw the edges connecting the $x_{i}$ as the "trunk" of an inverted tree; edges then branch off at each level, from left to right according to the order $\mathcal{O}$. The dynamics of $T_{\mathcal{O}}$ then simply sends an infinite vertical string to the next one to the right. See Fig. 1 for the odometer and Morse examples, and also see Figs. 8, 9, 10 below.

This figure also shows an orbit of the corresponding substitution dynamical system: it is the very bottom row of digits, and is shifted to the left by the same dynamics! Choosing as the trunk $x_{0} x_{1} \cdots=000 \ldots$, for the adic map this is the periodic sequence $.0101010 \ldots$, while for the Morse example it is the famous Morse-Thue sequence $.01101001 \ldots$ Taking the collection of all possible trunks, one can show we get the orbit closure space $\Omega$. This gives a different way of constructing $\Omega$ (and which easily generalizes to the nonstationary situation, where "fixed points" may not 
exist!) Now we can see the precise relationship between the adic transformation and the substitution dynamical system $(\Omega, S)$ : there is always a factor map from the adic transformation to the substitution dynamical system, and this is a bijection exactly when we can recover all the higher information in the tree from its "leaves" (the lowest level in the figure). This is possible for the Morse substitution as it satisfies Mosse's conditions of being primitive and recognizable [Mos92], [Mos96], [Hos00], giving Livshits' observation, but not for the odometer, where the substitution dynamical system consists of a single periodic orbit of period two. Thus the validity of the Livshits correspondence depends heavily on the particular order on the diagram. See the end of $\S 5$ for another concrete case of this.

As we have indicated, one can also make sense of the Livshits correspondence in the nonstationary setting. For an example of this, studied in [AF01], we define a pair of substitutions $\rho_{a}, \rho_{b}$ with constant alphabet $\mathcal{A}=\{0,1\}$ by $\rho_{a}(0)=0, \rho_{a}(1)=10$ and $\rho_{b}(0)=01, \rho_{b}(1)=1$. Choosing a sequence $\underline{n}=\left(. n_{0} n_{1} \ldots\right)$ in $\Pi_{0}^{\infty} \mathbb{N}^{*}$ where $\mathbb{N}^{*}=\{1,2, \ldots\}$, we define a substitution sequence $\left(\eta_{i}\right)_{i \geq 0}$ by $\eta_{i}=\rho_{a}$ for $0 \leq i \leq n_{0}-1$, $\eta_{i}=\rho_{b}$ for $n_{0} \leq i \leq n_{0}+n_{1}-1$, and so on. Note that the associated matrices are $M_{a}=\left[\begin{array}{ll}1 & 1 \\ 0 & 1\end{array}\right], M_{b}=\left[\begin{array}{ll}1 & 0 \\ 1 & 1\end{array}\right]$.

One can then prove that the corresponding nonstationary substitution dynamical system (respectively adic transformation) is minimal and uniquely ergodic, and that both are measure-theoretically isomorphic to the circle rotation $x \mapsto x+\theta(\bmod 1)$ of angle $\theta=\alpha /(1+\alpha) \in(0,1 / 2)$, where

$$
\alpha=\left[n_{0} \ldots n_{k} \ldots\right] \equiv \frac{1}{n_{0}+\frac{1}{n_{1}+\cdots}} .
$$

To get the angles in $(1 / 2,1)$ we begin with $\rho_{b}$ instead of $\rho_{a}$. See [AF01] for the nonstationary substitution point of view and [Fis08] for the adic version of this.

An interesting feature of this model is that $\# \mathcal{N S}=1$ while $\# \mathcal{N} \mathcal{P}=2$; as a consequence there is no possible extension of $T$ to a homeomorphism on the whole space. On the other hand, a different "dual" definition of the substitutions (with the same matrices) does permit such an extension, which topologically factors onto the circle rotation. See [Fis08] and [Fis] for a geometrical explanation of what we mean by duality and why this phenomenon occurs.

This representation of circle rotations is the simplest example of an adic model for interval exchange transformations (as a circle rotation is an exchange of two intervals); that such a model is possible is implicit in [Fer97], where it is shown that every interval exchange can be naturally represented as a cutting and stacking transformation, while each cutting and stacking transformation has an adic model. A precise realization based on the Rauzy induction procedure is presented in [Fis]; for minimal interval exchange transformations the map from the adic transformation to the interval exchange is almost surely bijective.

The difference between the stationary and nonstationary examples we have described is highlighted by bringing in the associated shift dynamics. For the odometer and Morse examples, the adic transformations are transverse dynamical systems to 
the left shift map $\left(\Sigma_{M}^{+}, \sigma\right)$; they satisfy a similar commutation relation, $\sigma \circ T^{2}=T \circ \sigma$ to that for the the stable horocycle flow and geodesic flow on a compact surface of constant negative curvature, for which $h_{s} \circ g_{t}=g_{t} \circ h_{e^{t} s}$. See [Ver94] regarding the general philosophy of transverse dynamics and more examples.

Note that since in the above equation $T$ is semiconjugated to its square, it must have entropy equal to either 0 or $\infty$. And indeed, for the Morse and odometer examples, both maps are zero entropy, minimal and uniquely ergodic; this is an immediate consequence of the fact that the maps permute the collection of cylinder sets of each level. These three properties also hold for the horocycle flow, see [Fur73], and for all stationary adic transformations with primitive matrix as we explain shortly.

Similarly, a general adic transformation $\left(\Sigma_{(M)}^{+, 0}, T_{\mathcal{O}}\right)$ can be thought of as a transverse dynamical system, with respect to the nonstationary dynamics of the left shift map on the nsft $\left(\Sigma_{(M)}^{+}, \sigma\right)$. Considering for example the circle rotation adic transformations, in fact all of this can be imbedded in an appropriate flow space, which extends Veech's Teichmüller flow, here for the simplest (torus) case, see[AF01].

In this nonstationary shift case, however, the three properties of zero entropy, minimality and uniquely ergodicity may no longer all hold true; one can have minimality without unique ergodicity, as described below; also the transverse map may have entropy in $(0, \infty)$; indeed by Vershik's general theorem, all ergodic maps have adic models; we note that in the positive entropy case the alphabet sizes must however be unbounded.

Now we turn to the criteria for minimality and unique ergodicity we shall use below.

Considering first the stationary case, as shown in Lemma 2.4 of [BM77], for an sft $\Sigma_{M}^{+}$with $M$ primitive, then the stable equivalence relation on $\left(\Sigma_{M}^{+}, \sigma\right)$ is uniquely ergodic. At the time the Bowen-Marcus paper was written, adic transformations had not yet been defined, but in this alternative language their theorem says that any adic transformation $T$ defined on $\Sigma_{M}^{+}$with $M$ primitive is uniquely ergodic. The BowenMarcus proof has these ingredients: first, primitivity of $M$ implies the shift map is mixing for invariant Markov measures whose support is the sft, hence for the Parry measure $\mu$ (the measure of maximal entropy), see Theorem 1.31 of [Wal82]; next, using mixing, there is a unique transverse invariant probability measure $\nu$ equivalent to this (i.e. sharing the same null sets); this is the eigenmeasure for the Ruelle operator with potential zero.

In [Fis08] we extend this proof to the nonstationary context as follows. First, we define a matrix sequence $\left(M_{i}\right)_{i \geq 0}$ to be primitive iff for each $k \in \mathbb{N}$, there exists $m>0$ (depending on $k$ ) such that all entries of the matrix $M^{(k, m)} \equiv M_{k} M_{k+1} \ldots M_{m-1}$ are strictly positive. We say the sequence is Perron-Frobenius iff the images of the positive cone $C_{m}$ in the space of column vectors $\mathbb{R}^{l_{m}}$ by the matrices when applied in the following order, $M_{k} M_{k+1} \ldots M_{m-1} C_{m}=M^{(k, m)} C_{m}$, nest down to a single strictly positive ray. Next we define nonstationary Parry measure (done in the last section of [AF01] for the special case of rotation adics), and extend mixing to the nonstationary context in an appropriate way. Then, making use of the projective metric, we show the Perron-Frobenius condition is equivalent to nonstationary mixing, and lastly we push through a Bowen-Marcus type of argument. Our conclusion is that for a primitive sequence $\left(M_{i}\right)_{i \geq 0}$, unique ergodicity is equivalent to the Perron-Frobenius condition. 
The primitivity condition was introduced by Livshits and Vershik in the fundamental paper [LV92], where it was remarked that primitivity of the sequence implies minimality of the adic transformation; the (simple) proof is given in [Fis08]. No doubt due to this fact, Livshits and Vershik choose this term ("minimality") for what we prefer to call primitivity; one reason for our choice of terminology is that there are examples of minimal adic transformations with nonprimitive sequences, as shown below by the Chacon example.

A first example of an adic transformation which is minimal but not uniquely ergodic is the Pascal adic transformation defined by Lodkin and Vershik [LV85], so-called because the Bratteli diagram is like the Pascal triangle laid over on its side, with $\# A_{n}=n$ for $n \geq 0$ and matrices $M_{n}$ with $\left(M_{n}\right)_{i j}=1$ if $j=i, i+1$ and otherwise $=0$. This map in fact has a continuum of invariant measures, indexed by the collection of Bernoulli coin-tossing measures; for an in-depth study see [MP05]. However we shall concentrate here on a very different type of example, which has a bounded alphabet size.

Our examples are closest in spirit to Keane's interval exchange. In fact any exchange of $d$ intervals codes in a natural way as an adic transformation on an alphabet of $d$ symbols, with the matrices being those that naturally appear in the theory for a quite different purpose (that of describing the induction procedure); see [Fer97] and [Fis]. The matrices occuring in Keane's famous counterexample give a $(4 \times 4)$ nonnegative unimodular (i.e. determinant one), primitive matrix sequence $\left(M_{i}\right)_{i \geq 0}$ for which the Perron-Frobenius property fails, so by our criterion the adic transformation is minimal but not uniquely ergodic as is the case for the interval exchange.

It is known that Keane's counterexample has the least number of intervals possible (four) for such a map; that of Keynes and Newton had five intervals. So the question we asked ourselves is: within the much wider realm of adic transformations, what is the smallest alphabet possible for an "adic counterexample", first, if we require unimodularity; next, if we remove that restriction? We show:

Theorem. For the unimodular case, there are $(3 \times 3)$ counterexamples, but no such $(2 \times 2)$ adic transformation is possible. Without the restriction of determinant one, there are $(2 \times 2)$ counterexamples as well.

We next turn to the Chacon example. This is a stationary adic transformation with nonunimodular matrix $A=\left[\begin{array}{ll}3 & 0 \\ 1 & 1\end{array}\right]$ which is nevertheless both minimal and uniquely ergodic. We call this the Chacon adic transformation because of its relation to a well-known substitution known by that name, see [Fer95].

For the primitive case unique ergodicity is true equivalently for the adic transformation, for any extension, and for the group $\mathcal{F C}$; for the nonprimitive case this may no longer quite be true, as shown by this example. Here minimality and unique ergodicity hold for the adic transformation, but what is true for $\mathcal{F C}$ is that the action is essentially minimal and essentially uniquely ergodic as defined above. We then show that despite the nonprimitivity, minimality and unique ergodicity remain true for any 
other adic transformation with this same matrix. See Proposition 5.2. In fact for this example $\mathcal{N}$ is a single point, which is a fixed point for $\mathcal{F C}$, and there is no continuous extension of the adic transformation to this point (see Remark 5.1).

Our proof of essential minimality and unique ergodicity for the Chacon adic goes by way of coding to a $(3 \times 3)$ primitive example. The proof is geometrical and is based on the stable tree view of the adic transformations explained above. We mention that though this is a coding from one subshift of finite type space to another, it has the interesting feature that though it conjugates the adic transformations, it does not (quite) conjugate the corrresponding shift maps.

Further remarks. For alphabet size one, the nontrivial examples are always both minimal and uniquely ergodic. Indeed, the matrix sequence is then $M_{i}=\left[n_{i}\right]$ for some integers $n_{i}$, with $n_{i} \geq 2$ infinitely often to avoid a trivial path space; there is up to permutation only one incoming edge order possible, so there is a single adic transformation compatible with each such sequence. This is a nonstationary odometer in that it is a nonstationary version of the Kakutani-von Neumann $d$-adic odometer (or adding machine), given by constant case $n_{i}=d$. The map permutes the cylinder sets of each level, and so is both minimal and uniquely ergodic, the only possible invariant probability measure giving equal mass to each cylinder. (Alternatively, the $(1 \times 1)$ matrix sequence is trivially both primitive and Perron-Frobenius, so minimality and unique ergodicity follow by our criteria.)

If one wants to prove that the Perron-Frobenius property holds for a given matrix sequence, then a useful tool is Birkhoff's upper bound for the projective metric, see [Fis08]. To produce a counterexample, however, we need a lower bound and one needs to consider more closely the combinatorics of the matrix sequence. This is the kind of argument that occurs in [Kea75] and which we encounter here.

For background on interval exchanges see [Kea75], [Kea77], [Vee78], [Vee82], [Mas82] and the recent excellent exposition [Via06]. Regarding substitution dynamical systems see [Fer02], [Fer95], [Hos00] and regarding adic transformations, see [LV92], [Ver89], [Ver94], [Ver95b], [Ver95a].

After this paper and [Fis08] had been submitted we received the preprint [BKMS09]; despite its title and abstract, in fact the nonstationary case is also addressed, see Theorem 2.8 there, and in particular the authors have independently come up with a completely different proof from that of [Fis08] that the Perron-Frobenius property implies unique ergodicity. In fact their argument is related to the "column stochastic matrix" method used in the study below of the nonsymmetric $(2 \times 2)$ case, as both can be understood via a cutting-and-stacking construction of the invariant measures; this argument can be extended to the general primitive as well as certain nonprimitive cases, including the Chacon example and the infinite measure example of [Fis92], see in particular Remark 2.10 of [BKMS09] regarding the infinite measure case.

Acknowledgements: We thank Xavier Bressaud and Julien Cassaigne for suggesting we consider the $(2 \times 2)$ counterexample and Chacon substitutions respectively, and Thierry Monteil as well as Cassaigne for conversations about the Chacon map. 
We thank B. Solomyak for his suggestion that the Birkhoff contraction bounds given in [Fis08] might furnish a converse to the statement of Proposition 3.1; we have included this argument in the final version of the paper.

\section{The unimodular $(2 \times 2)$ CASE}

First we show that a $(2 \times 2)$ unimodular "adic Keane counterexample" cannot exist.

Definition 2.1. We say an adic transformation is nontrivial if the space $\Sigma_{(M)}^{+, 0} \backslash \mathcal{N}$ on which the transformation acts is nonempty.

Proposition 2.1. Let $\left(M_{i}\right)_{i=0}^{\infty}$ for $i \geq 0$ be a sequence of $(2 \times 2)$ unimodular (i.e. determinant-one) nonnegative integer matrices. Then for any (nontrivial) adic transformation defined from the matrices, minimality is equivalent to unique ergodicity, and both are equivalent to primitivity of the matrix sequence.

Proof. The collection of nonnegative matrices with determinant one is the semigroup $S L(2, \mathbb{N})$ (here $\mathbb{N}=\{0,1, \ldots\})$, which is generated freely by the matrices $P=\left[\begin{array}{ll}1 & 0 \\ 1 & 1\end{array}\right]$ and $Q=\left[\begin{array}{ll}1 & 1 \\ 0 & 1\end{array}\right]$ (this is well known, for a proof see e.g. Lemma 3.11 of [AF05]). Given a sequence $\left(M_{i}\right)_{i=0}^{\infty}$ in $S L(2, \mathbb{N})$, we factor in this way each $M_{i}$ sucessively, producing a new sequence $\left(A_{j}\right)_{j=0}^{\infty}$ with each $A_{j}=P$ or $Q$. This additive sequence is clearly primitive if and only if $A_{j}$ is not eventually always equal to $P$ or $Q$. In that case, we can take partial products to produce a multiplicative sequence of the form $\widehat{A}_{i}=$ $\left[\begin{array}{cc}1 & 0 \\ n_{i} & 1\end{array}\right]$ or $\widehat{A}_{i}=\left[\begin{array}{cc}1 & n_{i} \\ 0 & 1\end{array}\right]$, for $n_{i}$ positive integers, with the choice of upper or lower triangular alternating for $i$ even or odd. Hence in $\left(A_{i}\right)$ the pair $A_{i} A_{i+1}=Q P=\left[\begin{array}{ll}2 & 1 \\ 1 & 1\end{array}\right]$ occurs infinitely often; a simple direct argument then shows the cones $M_{0} M_{1} \cdots M_{k} C^{+}$ nest down to a single direction, proving the topological Perron-Frobenius condition. For a second proof note that $Q P$ gives a definite contraction in the projective metric and so the projective diameter of these cones goes to zero, see [Fis08]; for a third proof, Proposition 4.1 of [AF05] explicitly finds the unique positive right eigenvector sequence for the matrix sequence $\left(\widehat{A}_{i}\right)$, existence of which is equivalent to the PerronFrobenius condition.

Applying these criteria, we therefore have minimality and unique ergodicity in the case where infinitely many of both $P$ and $Q$ appear. And if on the other hand the sequence ends with infinitely many $P$ or $Q$, then the adic transformation is trivial, as $\mathcal{N}$ is the whole space.

From a different perspective, the adic transformation can in either case be extended in a unique way to all of $\Sigma_{(M)}^{+, 0}$; it factors onto an irrational circle rotation in the nontrivial case, and onto a finite periodic orbit in the trivial case. See the introduction and Examples 3, 4 of [Fis08]. 


\section{Nonunimodular $(2 \times 2)$ COUnterexamples}

3.1. A symmetric counterexample. We define a sequence of symmetric $(2 \times 2)$ matrices with determinant greater than one (in fact growing very rapidly) and give a condition for the sequence to be primitive but not Perron-Frobenius. These are $M_{i}=\left[\begin{array}{cc}n_{i} & 1 \\ 1 & n_{i}\end{array}\right]$, for $\left(n_{i}\right)$ a sequence of positive integers. Our result is:

Proposition 3.1. Any adic transformation with matrix sequence $\left(M_{i}\right)_{i \geq 0}$ is minimal; if $\sum_{i=0}^{\infty} 1 / n_{i}<\infty$, then it is not uniquely ergodic; if $\sum_{i=0}^{\infty} 1 / n_{i}=+\infty$ then it is uniquely ergodic.

Proof. The sequence $\left(M_{i}\right)_{i>0}$ is certainly primitive, implying minimality. For the first statement we show first that if $\sum_{i=0}^{\infty} 1 / n_{i}<1$, then it is not Perron-Frobenius. We write $M_{i}=n_{i} I+N$ where $I$ is the identity matrix and $N=\left[\begin{array}{ll}0 & 1 \\ 1 & 0\end{array}\right]$ is nilpotent (i.e. $\left.N^{2}=I\right)$. Now,

$$
M_{0} M_{1} \cdots M_{k}=\Pi_{i=0}^{k}\left(n_{i} I+N\right)=\left[\begin{array}{ll}
a_{k} & b_{k} \\
b_{k} & a_{k}
\end{array}\right]=a_{k} I+b_{k} N
$$

where $a_{0}=n_{0}, b_{0}=1$ and

$$
\begin{aligned}
& a_{k+1}=n_{k+1} a_{k}+b_{k}, \\
& b_{k+1}=a_{k}+n_{k+1} b_{k} .
\end{aligned}
$$

From

$$
\frac{b_{k+1}}{a_{k+1}}=\frac{a_{k}+n_{k+1} b_{k}}{n_{k+1} a_{k}+b_{k}} \leq \frac{a_{k}+n_{k+1} b_{k}}{n_{k+1} a_{k}}=\frac{b_{k}}{a_{k}}+\frac{1}{n_{k+1}},
$$

we prove by induction that for all $k \geq 0$

$$
\frac{b_{k}}{a_{k}} \leq \sum_{i=0}^{k} \frac{1}{n_{i}} .
$$

So if $\sum_{i=0}^{\infty} \frac{1}{n_{i}}=\alpha<1$, then

$$
\limsup _{k \rightarrow \infty} \frac{b_{k}}{a_{k}} \leq \alpha<1
$$

or equivalently:

$$
\liminf _{k \rightarrow \infty} \frac{a_{k}}{b_{k}} \geq \alpha^{-1}>1
$$

The image of the positive cone, $M_{0} M_{1} \cdots M_{k} C^{+}$, is a symmetric cone bounded below by the vector $\left[\begin{array}{ll}a_{k} & b_{k} \\ b_{k} & a_{k}\end{array}\right]\left[\begin{array}{l}1 \\ 0\end{array}\right]$ and above by $\left[\begin{array}{ll}a_{k} & b_{k} \\ b_{k} & a_{k}\end{array}\right]\left[\begin{array}{l}0 \\ 1\end{array}\right]$; these vectors have slope $\leq \alpha<1$ and $\geq \alpha^{-1}>1$ respectively, so the (nested) intersection of the cones also has these bounds, and is nontrivial. This proves that the Perron-Frobenius condition fails. 
Next, if $\sum_{i=0}^{\infty} 1 / n_{i}<\infty$, we simply apply the argument to the matrix sequence $\left(M_{i}\right)_{i \geq k}$ where $k$ is such that $\sum_{i=k}^{\infty} 1 / n_{i}<1$. Since by definition of the PerronFrobenius condition the cones must nest to a single ray for each $k \geq 0$, this proves the claim.

For the second statement we follow a suggestion of Boris Solomyak and make use of the contraction upper bounds given in [Fis08]. We know that the Perron-Frobenius condition is satisfied iff the Birkhoff contraction coefficient for the matrix product $M_{0} M_{1} \cdots M_{k}$ goes to zero; combining Corollary 7.9 and Proposition 7.3 of [Fis08], an upper bound is given by the product of the coefficients for the individual matrices, which is equal to

$$
\prod_{0}^{k} \tanh \left(\frac{\theta_{i}}{4}\right)
$$

where $\theta_{i}=\log \left(n_{i}^{2}\right)$. So defining $\varepsilon_{i}=2 /\left(\exp \left(\theta_{i} / 2\right)+1\right)$, then this goes to 0 iff $\sum_{0}^{\infty} \log \left(1-\varepsilon_{i}\right)=+\infty$, iff $\sum_{0}^{\infty} \varepsilon_{i}=+\infty$, iff $\sum_{0}^{\infty} \exp \left(-\theta_{i} / 2\right)=\sum_{i=0}^{\infty} 1 / n_{i}=+\infty$, as claimed.

3.2. A nonsymmetric counterexample. This class of examples has several motivations: to give a quite flexible and general criterion for a $(2 \times 2)$ sequence to be a counterexample, to introduce some techniques for dealing with this nonsymmetric case, and to examine in isolation combinatorics which arose in the study of related interval exchange counterexamples on four intervals [FZ06].

From a "purely adic" point of view, the intuition behind the choice of matrices is similar to that for the symmetric case. Given a matrix sequence $\left(M_{i}\right)_{i>0}$, if the determinant of $M_{0} \cdots M_{k}$ is strictly positive, then equivalently the cone $C_{k}^{+}=M_{0} \cdots M_{k} C^{+}$ is not a single ray. For a counterexample we want this in the limit, that the infinite intersection $\cap_{k} C_{k}^{+}$is also nontrivial; the rough idea is to require that for $k$ large $M_{k}$ be projectively sufficiently close to the identity that the cones decrease by a summable amount. For the symmetric case just treated we carried this out explicitly. But now we take a different approach, first normalizing the matrices: we replace $\left(M_{i}\right)_{i \geq 0}$ by a related stochastic matrix sequence $\left(P_{i}\right)_{i \geq 0}$. It is important for the proof that these are column-stochastic rather than the usual row-stochastic sequences which appear in Markov chain theory (also in the nonstationary setting, as in [Fis08]).

Our result is:

Theorem 3.2. For a nonnegative integer matrix sequence $\left(M_{k}\right)_{k \geq 0}$ satisfying the four conditions which follow, then any adic transformation with matrix sequence $\left(M_{k}\right)_{k \geq 0}$ is minimal but is not uniquely ergodic.

Proof. We write $M_{k}=\left[\begin{array}{cc}n_{k} & \bar{n}_{k} \\ m_{k} & \bar{m}_{k}\end{array}\right]$, with strictly positive integer entries. So this is primitive, and we are to show the sequence $\left(M_{k}\right)_{k \geq 0}$ is not Perron-Frobenius.

We set for $k \geq 0$,

$$
M_{0} M_{1} \cdots M_{k}=\left[\begin{array}{ll}
a_{k} & b_{k} \\
c_{k} & d_{k}
\end{array}\right]
$$


giving

$$
\begin{aligned}
& a_{k+1}=n_{k+1} a_{k}+m_{k+1} b_{k}, \\
& b_{k+1}=\bar{n}_{k+1} a_{k}+\bar{m}_{k+1} b_{k},
\end{aligned}
$$

with similar equations (which we shall not need) for $\left(c_{k}\right)$ and $\left(d_{k}\right)$.

\section{Conditions:}

[1] Let $\left(\lambda_{i}\right)_{i \geq 1},\left(\xi_{i}\right)_{i \geq 1}$ be real sequences $\geq 1$ such that

$$
\sum_{i \geq 1} \frac{1}{\lambda_{i}} \text { and } \sum_{i \geq 1} \frac{1}{\xi_{i}}<+\infty
$$

Defining

$$
K=\exp \left(\sum_{i \geq 1} \frac{1}{\lambda_{i}}+\sum_{i \geq 1} \frac{1}{\xi_{i}}\right)
$$

we then define $\lambda_{0}=\xi_{0}=K$.

We have two integer sequences $\bar{n}_{k}, m_{k} \geq 1$ for $k \geq 0$ (the sequences can be fixed from the beginning, or these values can be chosen along the way), and then choose inductively two further integer sequences $n_{k}, \bar{m}_{k} \geq 1$ so that these four sequences satisfy:

$[2]$

$$
n_{0} \bar{m}_{0}>\bar{n}_{0} m_{0}
$$

so equivalently, $\operatorname{det} M_{0}>0$,

and so that for all $k \geq 0$ :

$[4]$

$$
\begin{gathered}
\bar{m}_{k+1}>\bar{n}_{k+1}\left(\lambda_{k} \frac{n_{k}}{\bar{n}_{k}}\right), \\
n_{k+1}>m_{k+1}\left(\xi_{k} \frac{b_{k}}{a_{k}}\right) .
\end{gathered}
$$

Lemma 3.3. Conditions $[3]$ and $[4]$ guarantee that

and that for all $k \geq 1$,

$$
\frac{\alpha_{0}}{\beta_{0}} \equiv \frac{n_{1}}{\bar{n}_{1}} \times \frac{n_{0} \bar{n}_{1}+\bar{n}_{0} \bar{m}_{1}}{n_{0} n_{1}+\bar{n}_{0} m_{1}}>K
$$

$$
\operatorname{det} M_{k}=n_{k} \bar{m}_{k}-\bar{n}_{k} m_{k}>0
$$

Proof of Lemma 3.3. We start by proving [5]. From [3] and [4], for $k=0$, since $\lambda_{0}=\xi_{0}=K$, we have:

$$
\bar{m}_{1}>K \frac{n_{0}}{\bar{n}_{0}} \bar{n}_{1} \text {, and } m_{1}<\frac{n_{0}}{\bar{n}_{0} K} n_{1} .
$$

Hence

$$
\frac{\alpha_{0}}{\beta_{0}} \equiv \frac{n_{1}}{\bar{n}_{1}} \times \frac{n_{0} \bar{n}_{1}+\bar{n}_{0} \bar{m}_{1}}{n_{0} n_{1}+\bar{n}_{0} m_{1}}=\frac{n_{1}}{\bar{n}_{1}}\left(\frac{\bar{n}_{1}+\frac{\bar{n}_{0}}{n_{0}} \bar{m}_{1}}{n_{1}+\frac{\bar{n}_{0}}{n_{0}} m_{1}}\right)>\frac{1+K}{1+\frac{1}{K}}=K .
$$


We next prove [6]. Multiplying [3] and [4] gives for all $k \geq 0$

$$
n_{k+1} \bar{m}_{k+1}>\bar{n}_{k+1} m_{k+1}\left(\lambda_{k} \xi_{k} \frac{n_{k}}{\bar{n}_{k}} \frac{b_{k}}{a_{k}}\right) \geq \bar{n}_{k+1} m_{k+1}\left(\frac{n_{k}}{\bar{n}_{k}} \frac{b_{k}}{a_{k}}\right) .
$$

So if we can show that

$$
\frac{n_{k}}{\bar{n}_{k}} \frac{b_{k}}{a_{k}} \geq 1
$$

then indeed $\operatorname{det} M_{k+1}>0$ for any $k \geq 0$. We prove (3) by induction. Recalling that $a_{0}=n_{0}$ and that $b_{0}=\bar{n}_{0}$, it is true for $k=0$. Assuming (3) holds for $k$ we prove it for $(k+1)$. To this end, we define the following function on the reals:

$$
f_{k}(t)=\frac{\bar{n}_{k}+\bar{m}_{k} t}{n_{k}+m_{k} t} .
$$

Its derivative is

$$
f_{k}^{\prime}(t)=\frac{\operatorname{det} M_{k}}{\left(m_{k} t+n_{k}\right)^{2}}
$$

(indeed $f_{k}$ is a fractional linear transformation!). By the induction hypothesis this is $>0$ so $f_{k}(\cdot)$ is a strictly increasing function. Since $b_{k-1} / a_{k-1}>0$ for all $k \geq 1$, we arrive at

$$
\frac{b_{k}}{a_{k}}=f_{k}\left(\frac{b_{k-1}}{a_{k-1}}\right)>f_{k}(0)=\frac{\bar{n}_{k}}{n_{k}},
$$

which delivers (3) and hence $[6]$, as desired.

Proof of Theorem 3.2: We define an associated sequence $\left(P_{k}\right)_{k \geq 0}$ which is columnstochastic, that is, the column sums are one. This sequence of matrices will be easier to work with than the original sequence $\left(M_{k}\right)$. Choosing the initial row vector $\mathbf{h}_{-1}^{t}=\left[\begin{array}{ll}1 & 0\end{array}\right]$, we define $\mathbf{h}_{k}^{t}=\mathbf{h}_{-1}^{t} M_{0} M_{1} \cdots M_{k}=\left[\begin{array}{ll}a_{k} & b_{k}\end{array}\right]$; this is a left eigenvector sequence with eigenvalue one, i.e.

$$
\mathbf{h}_{k}^{t}=\mathbf{h}_{k-1}^{t} M_{k} \text { for } k \geq 1 ;
$$

the entries of $\mathbf{h}_{k}^{t}$ are strictly positive for $k \geq 0$.

We then write $H_{k}, k \geq 0$, for the diagonal matrix with entries given by $\mathbf{h}_{k}^{t}$, so

$$
H_{k}=\left[\begin{array}{cc}
a_{k} & 0 \\
0 & b_{k}
\end{array}\right]
$$

and we define our new matrix sequence by

$$
P_{k}=H_{k} M_{k+1} H_{k+1}^{-1}, \quad k \geq 0 .
$$

Writing $\mathbf{1}^{t}$ for the row vector

$$
\mathbf{1}^{t}=\left[\begin{array}{ll}
1 & 1
\end{array}\right]
$$

we note that

$$
\mathbf{1}^{t} P_{k}=\mathbf{1}^{t} H_{k} M_{k+1} H_{k+1}^{-1}=\mathbf{h}_{k}^{t} M_{k+1} H_{k+1}^{-1}=\mathbf{h}_{k+1}^{t} H_{k+1}^{-1}=\mathbf{1}^{t} .
$$

Thus $\mathbf{1}^{t}$ defines a (constant) left eigenvector for $P_{k}$ with eigenvalue one, so the $P_{k}$ are column-stochastic (with strictly positive entries). 
We write $P_{k}=\left[\begin{array}{cc}p_{k} & \bar{p}_{k} \\ 1-p_{k} & 1-\bar{p}_{k}\end{array}\right] ;$ then

$$
P_{0} P_{1} \cdots P_{k}=H_{0} M_{1} \cdots M_{k+1} H_{k+1}^{-1} \equiv\left[\begin{array}{cc}
\alpha_{k} & \beta_{k} \\
1-\alpha_{k} & 1-\beta_{k}
\end{array}\right]
$$

which is also column-stochastic. (One checks that for $k=0$ the previous definition of $\alpha_{0} / \beta_{0}$ in Lemma 3.3 agrees with this.) We now explain the strategy of the proof. Letting $C^{+}$denote the positive cone in the space of column vectors $\mathbb{R}^{2}$, then $C_{k}^{+}=$ $P_{0} P_{1} \cdots P_{k} C^{+}$is the cone spanned by the two column vectors

$$
\left[\begin{array}{c}
\alpha_{k} \\
1-\alpha_{k}
\end{array}\right] \text { and }\left[\begin{array}{c}
\beta_{k} \\
1-\beta_{k}
\end{array}\right]
$$

We wish to show that $C_{\infty}^{+}=\cap_{k \geq 0} C_{k}^{+}$is nontrivial.

By [6] of Lemma 3.3, $\operatorname{det} M_{1} M_{2} \cdots M_{k+1}>0$; this is equivalent to $\operatorname{det} P_{0} P_{1} \cdots P_{k}=$ $\alpha_{k}-\beta_{k}>0$, so $\beta_{k}<\alpha_{k}$ for all $k \geq 0$.

We claim that $\left(\alpha_{k}\right)$ is a decreasing and $\left(\beta_{k}\right)$ an increasing sequence. Indeed, $\beta_{k+1}=$ $\beta_{k}+\left(\alpha_{k}-\beta_{k}\right) \bar{p}_{k+1}$ with $\beta_{k}<\alpha_{k}$ and $\bar{p}_{k}>0$ for all $k \geq 0$; the argument for $\left(\alpha_{k}\right)$ is similar.

We write $\alpha=\lim _{k \rightarrow \infty} \alpha_{k}=\inf _{k \geq 0} \alpha_{k}$ and $\beta=\lim _{k \rightarrow \infty} \beta_{k}=\sup _{k \geq 0} \beta_{k}$. Note that $0<\alpha \leq \beta<1$. Therefore,

$$
\lim _{k \rightarrow \infty} P_{0} \cdots P_{k}=\lim _{k \rightarrow \infty}\left[\begin{array}{cc}
\alpha_{k} & \beta_{k} \\
1-\alpha_{k} & 1-\beta_{k}
\end{array}\right]=\left[\begin{array}{cc}
\alpha & \beta \\
1-\alpha & 1-\beta
\end{array}\right] .
$$

Now the cone $C_{\infty}^{+}$is spanned by the columns of this limiting matrix. We claim that it is nontrivial if and only if the cone $\cap_{k \geq 0} M_{0} M_{1} \ldots M_{k} C^{+}$is.

Indeed, $M_{0} M_{1} \ldots M_{k+1} C^{+}=M_{0} H_{0}^{-1} P_{0} \ldots P_{k} H_{k+1} C^{+}=M_{0} H_{0}^{-1} P_{0} \cdots P_{k} C^{+}$, since $H_{k+1} C^{+}=C^{+}$. So $\cap_{k \geq 0} M_{0} M_{1} \ldots M_{k+1} C^{+}=\cap_{k \geq 0} M_{0} H_{0}^{-1} C_{k}^{+}=M_{0} H_{0}^{-1} C_{\infty}^{+}$.

As a result, $C_{\infty}^{+}$is nontrivial if and only if $\beta<\alpha$.

The rest of the proof of Theorem 3.2 is devoted to showing that $\beta<\alpha$.

To this end, we define

$$
\left[\begin{array}{cc}
A_{k+1} & B_{k+1} \\
C_{k+1} & D_{k+1}
\end{array}\right]=P_{0} P_{1} \cdots P_{k} H_{k+1}=\left(P_{0} P_{1} \cdots P_{k-1} H_{k}\right) M_{k+1},
$$

so for $k \geq 1$, just as in (2),

$$
\begin{aligned}
& A_{k+1}=n_{k+1} A_{k}+m_{k+1} B_{k} \\
& B_{k+1}=\bar{n}_{k+1} A_{k}+\bar{m}_{k+1} B_{k}
\end{aligned} .
$$

On the other hand, we have:

So

$$
\left[\begin{array}{ll}
A_{k+1} & B_{k+1} \\
C_{k+1} & D_{k+1}
\end{array}\right]=P_{0} \cdots P_{k} H_{k+1}=\left[\begin{array}{cc}
\alpha_{k} & \beta_{k} \\
1-\alpha_{k} & 1-\beta_{k}
\end{array}\right]\left[\begin{array}{cc}
a_{k+1} & 0 \\
0 & b_{k+1}
\end{array}\right]
$$

$$
A_{k+1}=\alpha_{k} a_{k+1}, \text { and } B_{k+1}=\beta_{k} b_{k+1} \text {. }
$$


From the second equation in (4),

$$
A_{k}=\frac{B_{k+1}-B_{k} \bar{m}_{k+1}}{\bar{n}_{k+1}}
$$

Substituting into the first equation in $(4)$ for $k$ and $(k+1)$ gives

$$
\frac{B_{k+2}-B_{k+1} \bar{m}_{k+2}}{\bar{n}_{k+2}}=\frac{B_{k+1}-B_{k} \bar{m}_{k+1}}{\bar{n}_{k+1}} \cdot n_{k+1}+B_{k} m_{k+1}
$$

so

$$
\frac{B_{k+2}}{\bar{n}_{k+2}}+\left(\frac{n_{k+1} \bar{m}_{k+1}}{\bar{n}_{k+1}}-m_{k+1}\right) B_{k}=\left(\frac{\bar{m}_{k+2}}{\bar{n}_{k+2}}+\frac{n_{k+1}}{\bar{n}_{k+1}}\right) B_{k+1} .
$$

By $[6], \operatorname{det} M_{k+1}>0$ so the quantity in parentheses on the left is $>0$. Therefore, for all $k \geq 0$,

$$
B_{k+2}<\left(\bar{m}_{k+2}+\frac{n_{k+1} \bar{n}_{k+2}}{\bar{n}_{k+1}}\right) B_{k+1}
$$

Now from (2), $b_{k+1}>\bar{m}_{k+1} b_{k}$ and $\beta_{k}=\frac{B_{k+1}}{b_{k+1}}$ from (5).

This in conjunction with (6) (with index lowered by one) together with [3] leads to

$$
\beta_{k}<\frac{\bar{m}_{k+1}+\frac{n_{k} \bar{n}_{k+1}}{\bar{n}_{k}}}{\bar{m}_{k+1}} \frac{B_{k}}{b_{k}}=\left(1+\frac{n_{k} \bar{n}_{k+1}}{\bar{n}_{k} \bar{m}_{k+1}}\right) \beta_{k-1} \leq\left(1+\frac{1}{\lambda_{k}}\right) \beta_{k-1} .
$$

Thus

$$
\beta_{k}<\beta_{0} \prod_{i=1}^{k}\left(1+\frac{1}{\lambda_{i}}\right), k \geq 1
$$

We next find a lower bound for $\alpha_{k}$, and equivalently an upper bound for $1 / \alpha_{k}$. From (5) and (4), $\alpha_{k} a_{k+1}=A_{k+1}>A_{k} n_{k+1}=\alpha_{k-1} a_{k} n_{k+1}$ so, using the first equation in (2),

$$
\frac{1}{\alpha_{k}}<\frac{a_{k+1}}{a_{k}} \frac{1}{n_{k+1}} \frac{1}{\alpha_{k-1}}=\left(1+\frac{b_{k}}{a_{k}} \frac{m_{k+1}}{n_{k+1}}\right) \frac{1}{\alpha_{k-1}}<\left(1+\frac{1}{\xi_{k}}\right) \frac{1}{\alpha_{k-1}} .
$$

Therefore, for all $k \geq 1$,

$$
\frac{1}{\alpha_{k}}<\frac{1}{\alpha_{0}} \prod_{i=1}^{k}\left(1+\frac{1}{\xi_{i}}\right)
$$

Thus, multiplying (7) and (8) then taking the limit as $k \rightarrow \infty$, we arrive at

$$
\frac{\beta}{\alpha} \leq \frac{\beta_{0}}{\alpha_{0}} \prod_{i=1}^{\infty}\left(1+\frac{1}{\lambda_{i}}\right)\left(1+\frac{1}{\xi_{i}}\right) \leq \frac{\beta_{0}}{\alpha_{0}} \exp \left(\sum_{i=1}^{\infty} \frac{1}{\lambda_{i}}+\sum_{i=1}^{\infty} \frac{1}{\xi_{i}}\right)=\frac{\beta_{0}}{\alpha_{0}} K<1,
$$

where we have used [1] in deriving the first inequality, then the fact that $\log (1+x) \leq x$ for all $x>-1$ in writing the second one then finally condition [5]. So $\beta<\alpha$ and we are done with the proof of Theorem 3.2. 


\section{A $(3 \times 3)$ UNimOdULAR COUNTEREXAMPLE}

This class of examples is inspired by the combinatorics of the $(4 \times 4)$ matrices

$$
L_{j}=\left(\begin{array}{cccc}
0 & 0 & 1 & 1 \\
m_{j}-1 & m & 0 & 0 \\
n_{j} & n_{j} & n_{j}-1 & n_{j} \\
1 & 1 & 1 & 1
\end{array}\right)
$$

which appear in [Kea77]; they can be used to define an adic transformation which is isomorphic to the interval exchange, see [Fis] (though these matrices play a different role in Keane's paper).

The idea is to remove a row and column from the above matrices, following this by a permutation of the columns, with everything chosen in such a way as to allow the rest of Keane's argument to still go through.

Our matrix sequence $\left(M_{i}\right)_{i \geq 0}$ is:

$$
M_{j}=\left(\begin{array}{ccc}
m_{j} & 0 & m_{j}-1 \\
n_{j} & n_{j}-1 & n_{j} \\
1 & 1 & 1
\end{array}\right) .
$$

As in the last case $\operatorname{det} M_{j}=-1$, and again we replace it by the sequence $\left(M_{2 j} M_{2 j+1}\right)_{j \geq 0}$ to get a sequence with determinant one.

On $\mathbb{R}^{3}$ we use the norm $|\mathbf{x}|=\sum_{i=1}^{3}\left|x_{i}\right|$. Writing $C^{+}$for the positive cone of $\mathbb{R}^{3}$, then $C^{+}$projects to the unit simplex $\Delta=\left\{\mathbf{x} \in C^{+}:|\mathbf{x}|=1\right\}$ by $\pi: \mathbf{x} \mapsto \mathbf{x} /|\mathbf{x}|$. We normalize the linear map $M_{j}$ on the cone to a nonlinear map $\widetilde{M}_{j}$ on the simplex by $\widetilde{M}_{j}(\mathbf{x})=\pi \circ M_{j}(\mathbf{x})=M_{j}(\mathbf{x}) /\left|M_{j}(\mathbf{x})\right|$.

Theorem 4.1. Suppose that for $i \geq 0, m_{i}, n_{i}$ are positive integers satisfying: $n_{0} \geq 6$ and that for all $k \geq 0$,

$$
\left(3 n_{k}+1\right) \leq 2 m_{k} \leq n_{k+1} .
$$

Then the sequence $\left(M_{i}\right)_{i \geq 0}$ is not Perron-Frobenius, and so any adic transformation defined by taking the $M_{i}$ as edge matrices and then fixing an incoming edge order, is minimal (since the sequence is primitive) but is not uniquely ergodic.

First we need some lemmas. Fixing $k$, and given a vector $\mathbf{x}^{(k+1)} \in \Delta$, we define inductively for $0 \leq j \leq k$ :

$$
\mathbf{x}^{(j)}=\widetilde{M}_{j} \mathbf{x}^{(j+1)}
$$

so $\mathbf{x}^{(0)}=\widetilde{M}_{0} \circ \widetilde{M}_{1} \circ \cdots \circ \widetilde{M}_{k}\left(\mathbf{x}^{(k+1)}\right)$. What we will show is that there are two disjoint subsimplices in $\Delta$ such that for any $k,\left(\underline{e}_{1}\right)^{(0)}$ and $\left(\underline{e}_{2}\right)^{(0)}$ are in these subsimplices, see Fig. 2.

Lemma 4.2. For any $j \geq 0$, for any $\mathbf{x} \in \Delta,\left(\widetilde{M}_{j}(\mathbf{x})\right)_{3} \leq 1 / n_{j}$.

Proof. We have $\left(M_{j} \mathbf{x}\right)_{3}=1$ while $\left|M_{j}(\mathbf{x})\right|=n_{j}+\left(m_{j}+1\right) x_{1}+m_{j} x_{3} \geq n_{j}$.

Lemma 4.3. Taking $\mathbf{x}^{(k+1)}=\underline{e}_{2}$, fixing $k \geq 1$, then for any $0 \leq j \leq k+1, x_{1}^{(j)} \leq$ $1 / n_{j}$. 


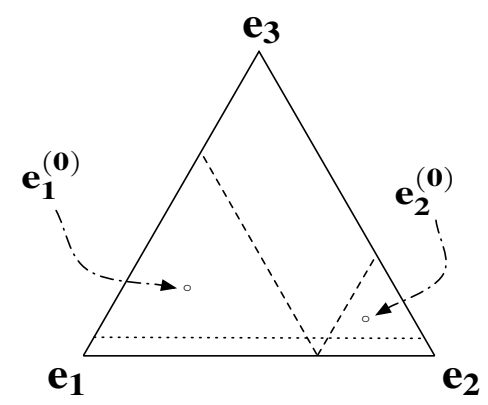

FiguRE 2. For each $k$, the image simplex $\Delta_{k}$ contains points which belong to disjoint subsimplices of $\Delta$.

Proof. For $(k+1)$ this is true since $x_{1}^{(k+1)}=\left(\underline{e}_{2}\right)_{1}=0$. For the (decreasing) induction step, assuming $x_{1}^{(j+1)} \leq 1 / n_{j+1}$ we shall prove that then $x_{1}^{(j)} \leq 1 / n_{j}$. Now we know that $x_{3}^{(j+1)} \leq 1 / n_{j+1}$ for any $j \leq k$; this is true for $j=k$ since $x_{3}^{(j+1)}=0$, while for all $j<k$ we apply Lemma 4.2. Now since

$$
x_{1}^{(j)}=\frac{m_{j} x_{1}^{(j+1)}+\left(m_{j}-1\right) x_{3}^{(j+1)}}{n_{j}+\left(m_{j}+1\right) x_{1}^{(j+1)}+m_{j} x_{3}^{(j+1)}}
$$

this will certainly be smaller than $1 / n_{j}$ if $m_{j} x_{1}^{(j+1)}+\left(m_{j}-1\right) x_{3}^{(j+1)} \leq 1$. And this follows from the condition $m_{j} \leq n_{j+1} / 2$ together with $x_{3}^{(j+1)}, x_{1}^{(j+1)} \leq 1 / n_{j+1}$.

Lemma 4.4. For $\mathbf{x}^{(k+1)}=\underline{e}_{2}$, for $k \geq 0, x_{2}^{(0)} \geq 1-2 / n_{0}$.

Proof. This follows from the previous two lemmas since $x_{2}^{(0)}=1-x_{1}^{(0)}-x_{3}^{(0)} \geq$ $1-\left(1 / n_{0}+1 / n_{0}\right)$.

Lemma 4.5. Taking now $\mathbf{x}^{(k+1)}=\underline{e}_{1}$, then for all $k \geq 0, x_{1}^{(0)}=\left(\underline{e}_{1}^{(0)}\right)_{1} \geq \frac{1}{3}$.

Proof. We prove in fact that for all $j \leq k+1, x_{1}^{(j)} \geq \frac{1}{3}$, again proceeding by induction. For $j=k+1, x_{1}^{(k+1)}=1 \geq \frac{1}{3}$; for the induction step we assume that $x_{1}^{(j+1)} \geq \frac{1}{3}$ and prove it for $j$.

Now again $x_{1}^{(j)}$ satisfies equation (9). We are to prove that

$$
3 m_{j} x_{1}^{(j+1)}+3\left(m_{j}-1\right) x_{3}^{(j+1)} \geq n_{j}+\left(m_{j}+1\right) x_{1}^{(j+1)}+m_{j} x_{3}^{(j+1)}
$$

or equivalently that

$$
\left(2 m_{j}-1\right) x_{1}^{(j+1)} \geq\left(3-2 m_{j}\right) x_{3}^{(j+1)}+n_{j}
$$

or

$$
2 m_{j}\left(x_{1}^{(j+1)}+x_{3}^{(j+1)}\right) \geq\left(x_{1}^{(j+1)}+x_{3}^{(j+1)}\right)+n_{j}+2 x_{3}^{(j+1)} .
$$

Using the assumption that $m_{j} \geq\left(3 n_{j}+1\right) / 2$, it is enough to show that

$$
3 n_{j}\left(x_{1}^{(j+1)}+x_{3}^{(j+1)}\right) \geq n_{j}+2 x_{3}^{(j+1)}
$$

or equivalently

$$
3 n_{j} x_{1}^{(j+1)} \geq n_{j}-\left(3 n_{j}-2\right) x_{3}^{(j+1)} .
$$


But since $x_{1}^{(j+1)} \geq \frac{1}{3}$ while $-\frac{1}{n_{j+1}} \leq-x_{3}^{(j+1)}$ (also for the initial case $x_{3}^{(k+1)}=0$ ) and $\left(3 n_{j}-2\right)>0$, this is true.

Proof of theorem 4.1: Since $M_{i}$ has entries all $>0$, the sequence $M_{i}$ is certainly primitive hence any adic transformation defined from it by choosing an incoming edge order on the Bratteli diagram is minimal.

But the image simplex $\Delta_{k} \equiv \widetilde{M}_{0} \circ \widetilde{M}_{1} \circ \cdots \circ \widetilde{M}_{k}(\Delta)$ contains the points $\underline{e}_{1}^{(0)}=$ $\underline{e}_{1}^{(0)}(k)$, with first coordinate $\geq 1 / 3$, and $\underline{e}_{2}^{(0)}=\underline{e}_{2}^{(0)}(k)$ whose second coordinate is $>1-2 / n_{1} \geq 2 / 3$; these conditions define two subsimplices which meet at a single point on the boundary of $\Delta$. By primitivity there exists $a>0$ such that the third coordinate of each is $>a$ for all $k$, so these points are in fact in disjoint subsimplices, see Fig. 2. Hence the image simplices $\Delta \supseteq \Delta_{1} \supseteq \cdots \Delta_{k+1} \cdots$ cannot nest down to a single point.

\section{A NONPRIMITIVE UNIQUELY ERGODIC ADIC TRANSFORMATION}

We call this example the Chacon adic transformation because of its connection to a substitution dynamical system of [Fer95], [Fer02], which in turn is closely related to Chacon's transformation, a well-known map of the unit interval constructed by cutting and stacking [Cha69]. The matrix which defines the Bratteli diagram is

$$
A=\left[\begin{array}{ll}
3 & 0 \\
1 & 1
\end{array}\right] \text {. }
$$

As described in the introduction, we think of the substitution $\rho: 0 \mapsto 0010, \rho: 1 \mapsto 1$ as mapping from right to left on the Bratteli diagram and so defining an incoming edge order, see Fig. 3.

We claim that both minimality and unique ergodicity hold for the adic transformation $\left(\Sigma_{A}^{+} \backslash \mathcal{N}, T_{\mathcal{O}}\right)$. We give a self-contained and "purely adic" proof of this, not making use of the substitution dynamical system (see Remark 5.2). In this proof we compare this map to two related adic transformations, also given by substitutions.

We call elements of the alphabet vertices and label edges by pairs of vertices separated by letters in $\{a, b, c, d\}$; a symbol is a vertex or a letter. To specify an edge path we could list the sequence of edges, but usually we write it in a shorter form as a single string of symbols, consisting of the corresponding vertex sequence with letters inserted when necessary to specify an edge unambiguously.

In the Chacon adic space $\Sigma_{A}^{+}$there are four incoming edges to the vertex 0 , ordered as $0 a 0<0 b 0<1 c 0<0 d 0$, and one edge 11 . We use a bar to indicate infinitely repeated symbols, so e.g. $(. \overline{0 d})=(.0 d 0 d \ldots)$. The set of points (i.e. edge paths) with no successor is $\mathcal{N} \mathcal{S}_{A}=\{(. \overline{0 d}),(. \overline{1})\}$ and those with no predecessor is $\mathcal{N} \mathcal{P}_{A}=$ $\{(. \overline{0 a}),(. \overline{1})\}$.

Remark 5.1. This gives an example of an adic transformation with no continuous extension to all of $\Sigma_{A}^{+}$. Indeed, no such definition is possible at the point $(. \overline{0 d})$, since the successor of $(.0 d 0 d \ldots 0 d 0 b 0)$ is $(.11 \ldots 10)$ while the successor of $(.0 d 0 d \ldots 0 d 0 a 0)$ is $(.0 a 0 a \ldots 0 a 0 b 0)$. 


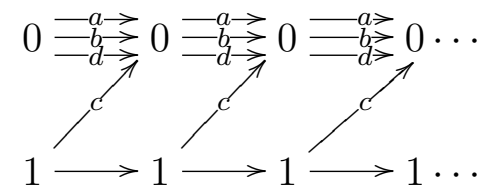

FiguRE 3. Bratteli diagram for the Chacon adic transformation; the edge paths form the space $\Sigma_{A}^{+}$; the substitution maps from right to left.

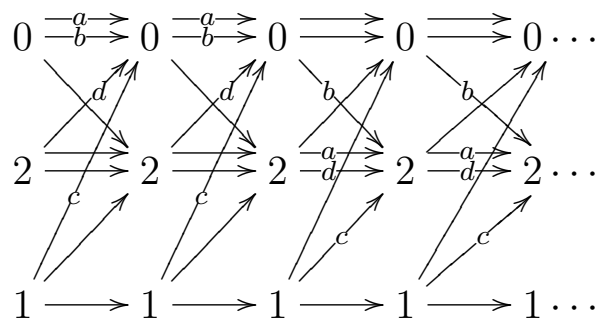

FIGURE 4. Diagram for the intermediate transformation, giving the space $\Sigma_{B}^{+}$; incoming orders for vertices 0 and 2 are shown.

Next we define two related stationary adic transformations, both with alphabet $\mathcal{A}=\{0,1,2\}$; see Figs. 4, 5. For the first of these, the intermediate transformation, the matrix is

$$
B=\left[\begin{array}{lll}
2 & 0 & 1 \\
1 & 1 & 1 \\
1 & 0 & 2
\end{array}\right] \text { with substitution } \rho_{B}: 0 \mapsto 0012,1 \mapsto 1,2 \mapsto 2012 .
$$

In this diagram there are 9 edges. Those entering vertex 0 are ordered $0 a 0<$ $0 b 0<1 c 0<2 d 0$ and those entering 1 are ordered $2 a 2<0 b 2<1 c 2<2 d 2$, and there is one edge 11 entering the vertex 1. Here $\mathcal{N S}_{B}=\{(. \overline{2 d}),(. \overline{1})\}$ and $\mathcal{N P}_{B}=$ $\{(. \overline{0 a}),(\overline{2 a}),(. \overline{1})\}$.

Next we define the final transformation, given by matrix

$$
C=\left[\begin{array}{lll}
2 & 0 & 1 \\
1 & 1 & 1 \\
1 & 1 & 1
\end{array}\right], \text { with substitution } \rho_{C}: 0 \mapsto 0012,1 \mapsto 12,2 \mapsto 012 .
$$

For this space, there are again 9 edges, with four entering vertex 0 ordered $0 a 0<$ $0 b 0<1 c 0<2 d 0$ (as before), then three entering 2 ordered as $0 b 2<1 c 2<2 d 2$ and lastly two entering 1 ordered by $11<2 a 1$. Now $\mathcal{N S}_{C}=\{(.2 d 2 d 2 d \ldots)\}$ and $\mathcal{N} \mathcal{P}_{C}=\{(.0 a 0 a 0 a \ldots),(.111 \ldots)\}$. We write $W^{s}((. \overline{1}))$ for the stable equivalence class of $(. \overline{1})$ : all sequences of the form $(. * * \cdots * 111 \ldots)$, where $*$ means "any symbol", and note that this is a countable set.

We note that the successor of $(. \overline{1})$ is now defined and equals $(.2 a \overline{1})$, which in turn maps to $(.0 b 2 a \overline{1})$, then $(.1 c 2 a \overline{1})$, and so on.

We shall prove: 


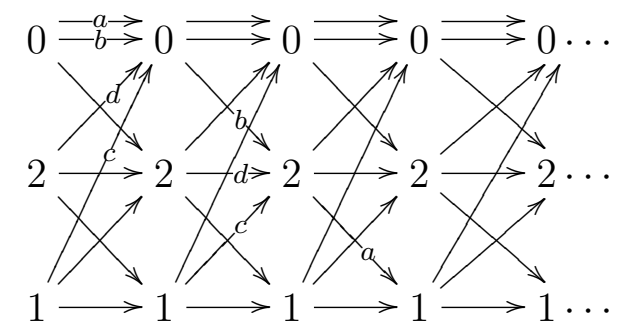

Figure 5. Diagram for the final transformation on $\Sigma_{C}^{+}$, showing incoming orders for vertices 0,2 and 1.

Theorem 5.1. There exist injective continuous maps $\Phi: \Sigma_{A}^{+} \rightarrow \Sigma_{B}^{+}$and $\Psi: \Sigma_{B}^{+} \rightarrow$ $\Sigma_{C}^{+}$, such that $\Phi$ is onto all of $\Sigma_{B}^{+}$minus a countable set and preserves the lexicographic orders, while $\Psi$ is a bijection, which is order-preserving after a single point has been removed from both spaces. The dynamics of the adic transformations $T_{A}$ on $\Sigma_{A}^{+} \backslash$ $\mathcal{N}_{A}, T_{B}$ on $\Sigma_{B}^{+} \backslash \mathcal{N}_{B}$ and $T_{C}$ on $\Sigma_{C}^{+} \backslash \mathcal{N}_{C}$ are conjugated by the maps. The shift transformations are also conjugated by $\Phi$, but this is not true for $\Psi$.

These maps conjugate the actions of $\mathcal{F C}_{A}$ restricted to $\Sigma_{A}^{+} \backslash\{. \overline{1}\}$, of $\mathcal{F C}_{B}$ on $\Sigma_{B}^{+} \backslash$ $\left(\{\overline{1}\} \cup W^{s}(. \overline{2 a})\right)$ and of $\mathcal{F C}_{B}$ on $\Sigma_{B}^{+} \backslash W^{s}(. \overline{1})$.

Proof. We define $\Phi: \Sigma_{A}^{+} \rightarrow \Sigma_{B}^{+}$.

Given $x=\left(x_{i}\right) \in \Sigma_{A}^{+}$, where the symbol sequence $x_{i} \in\{0,1, a, b, c, d\}$ specifies an edge path, we determine the image $\Phi(x)$ as follows. If we see a block consisting entirely of $0 d$ or $0 a$ and ending with $0 d$, we change each 0 to 2 . In particular, if the string $x$ ends in an infinite string of $0 a$ and $0 d$, which contains infinitely many $0 d$, then we change each 0 to 2 there.

We note that by this definition $\Phi$ is a well-defined map from $\Sigma_{A}^{+}$to $\Sigma_{B}^{+}$. It is also shift-invariant, since the code only depends on what happens to the right, so cutting off an edge on the left does not affect this. We claim next that $\Phi$ is in fact a bijection from $\Sigma_{A}^{+}$to $\Sigma_{B}^{+}$, and that it preserves lexicographic order. It will follow that $\Phi$ is a bijection on the sets $\mathcal{N S}, \mathcal{N} \mathcal{P}$ and $\mathcal{N}$ of the two spaces, and that it conjugates the successor maps $T_{A}: \Sigma_{A}^{+} \backslash \mathcal{N} \mathcal{S}_{A} \rightarrow \Sigma_{A}^{+} \backslash \mathcal{N} \mathcal{P}_{A}$ and $T_{B}: \Sigma_{B}^{+} \backslash \mathcal{N} \mathcal{S}_{B} \rightarrow \Sigma_{B}^{+} \backslash \mathcal{N} \mathcal{P}_{B}$ and hence the adic transformations on $\Sigma_{A}^{+} \backslash \mathcal{N}_{A}, \Sigma_{B}^{+} \backslash \mathcal{N}_{B}$.

For this one can give a combinatorial proof, checking all cases, but which contributes little to the understanding of what is going on. So instead we give a geometrical proof based on the pictures which underlie the combinatorial argument. These are pictures of infinite trees, which rather being infinite towards the branches as often occurs in mathematics are infinite towards the root (drawn upwards!), see Fig. 8. Each tree represents an equivalence class $W^{s}(x)$ for some $x=\left(x_{i}\right) \in \Sigma_{A}^{+}$; this is the stable tree of $x$, as described in the introduction. To construct the stable tree of a given string $x$, we write the vertices as nodes of the tree and the letters as edges, ordered from left to right; the $0^{\text {th }}$ vertex is at the bottom, the first above that, with the infinite trunk representing $x$ extending upwards and all the paths in $W^{s}(x)$ branching off to the left and right. The adic transformation then has a purely geometrical description, as shifting from right to left along the ending nodes at the bottom of this tree. 
Our map $\Phi$ is now easily described. It takes a stable tree in $\Sigma_{A}^{+}$to a stable tree in $\Sigma_{B}^{+}$as follows: we leave all the edges in place, but change some of the vertex labels from 0 to 2. The substitution maps in the downwards direction; this change is made so as to agree with the new substitution rule $\rho_{B}$, and leads to the coding description just given. This proves that we have a bijection which does preserve the lexicographic order, as claimed. The figure shows a finite part of a stable tree, ending on top with edge $0 b 0$. The situation depicted is typical for any such tree. Note that the code after (above) this location does not change this part of the code, and so this proof is valid no matter what are the previous symbols.

The figure shown covers only the case where some $0 b$ appears. There are thus two cases not covered. The first is where the string ends in $111 \ldots$ (which means it is necessarily $(.11 \ldots))$. Now $\Phi(.11 \ldots)=(.11 \ldots)$, handling that case. The second case is where it ends in a sequence of $0 a$ and $0 d$; this subdivides into the cases with finitely or infinitely many $0 d$. If there are finitely many $0 d$, then this is just like the figure but with a $0 a$ on the top replacing the $0 b$; nothing above that is changed. Lastly, if we have infinitely many $0 d$, then any subtree below a given $0 d$ behaves as if it were the $0 d$ branch below $0 b$ in the figure shown. The trees are nested as we go upwards, so the bijectivity and order-preservation hold here as well.

We have shown bijectivity and order-preservation from any stable tree in the first space to a corresponding tree in the second. To check surjectivity, we have to be sure that no stable tree in $\Sigma_{B}^{+}$has been entirely missed- but that is just what has happened! We have already covered all cases where 0 or where $2 d$ occurs infinitely often, so the only possibility is a string which ends in all $2 a$. And indeed, the path $(. \overline{2 a})$ has no preimage under $\Phi$. This point is in $\mathcal{N} \mathcal{P}_{B}$, and its stable tree is $W^{s}((. \overline{2 a}))=$ $\{(. \overline{2 a}),(.0 b \overline{2 a}),(.1 c \overline{2 a}), \ldots\}$, listed in dynamical order of $T_{B}^{n}((. \overline{2 a}))$.

We recall from $\S 2.2$ of [Fis08] that the group $\mathcal{F C}$ on the space $\Sigma_{A}^{+}$is generated by the collection of maps $\gamma$ which interchange two cylinder sets of equal length which end in the same edge. From the stable tree picture just described, we see that the cylinder sets in $\Sigma_{A}^{+}$ending with $0 d$ at some fixed level $n$ correspond bijectively to those in $\Sigma_{B}^{+}$ending on $2 d$, so these generating maps correspond via the conjugacy. The point $(. \overline{1})$ is a fixed point for $\mathcal{F C}$ in both spaces. Hence $\left(\Sigma_{A}^{+}, \mathcal{F C}_{A}\right)$ is conjugate to the restricted action $\left(\Sigma_{B}^{+} \backslash W^{s}(\overline{2 a}), \mathcal{F C}_{B}\right)$.

Next we define $\Psi: \Sigma_{B}^{+} \rightarrow \Sigma_{C}^{+}$.

We search in a string $y$ until we find the first occurence of $2 d 0$ or $2 d 2$. We code the maximal block $2 a 2 a \ldots 2 a 2 d 0$ to $2 a 1 \ldots 1 c 0$, and the maximal block $2 a 2 a \ldots 2 a 2 d 2$ to $2 a 1 \ldots 1 c 2$; in this definition we also allow no $a^{\prime} s$. We also code a maximal infinite string $\overline{2 a}$ which follows some finite string to $2 a \overline{1}$. All other strings are left unchanged. For some examples, $2 d 0$ is mapped to $1 c 0,2 a 2 a 2 a 2 d 0$ is mapped to $2 a 111 c 0$, and $(.1 c \overline{2 a 2 d})$ is mapped to $(.1 c \overline{2 a 1 c})$. We claim this defines an order-preserving map from $\Sigma_{B}^{+}$to $\Sigma_{C}^{+}$.

For the proof see Figs. 9, 10: we depict what happens for a finite tree below an edge $2 d 0$, the case of $2 d 2$ being similar. The map may remove an edge and replace it elsewhere, as indicated, but as the location in the tree is unchanged, this gives a bijection between the two finite stable trees, which moreover is order-preserving since 
the lexicographic orders have been represented geometrically from left to right. Note that the new tree faithfully represents the new substitution rule $\rho_{C}$.

The map on such a finite tree is well-defined, as knowing further edges above this will not change this part of the code (i.e. the tree pictures are nested). This implies that there is an order-preserving bijection between two stable trees when the string $y \in \Sigma_{B}^{+}$contains infinitely many vertices 0 or infinitely many occurences of $2 d$. The only other possibility in the domain of $\Psi$ is a string $(. \overline{1})$ or a string ending in $\overline{2 a}$. But by definition $\Psi(. \overline{1})=(. \overline{1})$ and the end of a string of the form $\overline{2 a}$ is coded to $2 a \overline{1}$. This concludes the proof that $\Psi$ is well-defined on all of $\Sigma_{B}^{+}$and is injective.

It remains to check surjectivity. Points of the following type have preimages, as we have seen: those with vertex 0 or $2 d$ occuring infinitely often. Also, any tree below $1 c 2$ has a well-defined preimage, as one sees from the figure. A path ending in $2 a \overline{1}$ is the image of one ending in $\overline{2 a}$, covering that case. The image of $(. \overline{1})$ is $(. \overline{1})$. This covers all cases, so the map is onto and hence is a bijection from $\Sigma_{B}^{+}$onto $\Sigma_{C}^{+}$.

To complete the verification of order-preservation there is one more tree to check in $\Sigma_{C}^{+}$, the tree containing $(.11 \ldots)$. In $\Sigma_{B}^{+}$this tree contains that one path but in $\Sigma_{C}^{+}$ the stable tree of $\Psi(.11 \ldots)=(.11 \ldots)$ has countably many paths. This string is in $\mathcal{N} \mathcal{S}_{B} \cap \mathcal{N} \mathcal{P}_{B}$ and in $\mathcal{N} \mathcal{P}_{C}$ but not $\mathcal{N} \mathcal{S}_{C}$, as there it has a successor, $\Psi(. \overline{2 a})=(.2 a \overline{1} \ldots)$. The adic orbit then follows the images of $T_{B}^{n}$ applied to this point, see Fig. 7. So $\Psi$ is an order-preserving bijection from $\Sigma_{B}^{+} \backslash\{(.11 \ldots)\}$ onto $\Sigma_{C}^{+} \backslash\{(.11 \ldots)\}$.

Interestingly, this time the map is not shift-invariant. For an example, $\Psi(.2 a 2 d 0 b 0)=$ $(.2 a 1 c 0 b 0)$ but $\Psi(\sigma(.2 a 2 d 0 b 0))=\Psi(.2 d 0 b 0)=(.2 d 0 b 0) \neq(.1 c 0 b 0)=\sigma(.2 a 1 c 0 b 0)$. One can also see this in the tree picture: whether or not one of the new branches is used depends on the final edge, so if this is removed no change is made.

Finally we consider the actions of $\mathcal{F C}_{B}$ and $\mathcal{F} \mathcal{C}_{C}$. By similar reasoning as before, the action of $\mathcal{F C}_{B}$ restricted to $\Sigma_{B}^{+} \backslash\left(W^{s}(. \overline{2 a}) \cup\{. \overline{1}\}\right)$ is conjugate to the action of $\mathcal{F C}_{C}$ on $\Sigma_{C}^{+} \backslash W^{s}(. \overline{1})$. The statement in the theorem follows.

We then conclude:

\section{Proposition 5.2.}

(i) The action of the group $\mathcal{F C}_{C}$ on $\Sigma_{C}^{+}$is minimal and uniquely ergodic; while this is false for $\mathcal{F C}_{A}$ on $\Sigma_{A}^{+}$and $\mathcal{F C}_{B}$ on $\Sigma_{B}^{+}$, both of these actions are essentially minimal and essentially uniquely ergodic.

(ii) Any adic transformation on $\left(\Sigma_{M}^{+} \backslash \mathcal{N}_{M}, T_{M, \mathcal{O}}\right)$ for $M=A, B$ or $C$ and for any order $\mathcal{O}$ is both minimal and uniquely ergodic.

Proof. (i) Since the matrix $C$ is primitive, by Lemma 2.4 of [BM77] the action of $\mathcal{F C}_{C}$ is uniquely ergodic. (Alternatively one can apply the criterion of [Fis08] to the constant sequence $C$, as the "Perron-Frobenius property" follows of course from the Perron-Frobenius theorem). Minimality follows easily from primitivity, as shown for the nonstationary case in [Fis08].

The invariant probability measure is nonatomic, and is taken by the conjugacies of Theorem 5.1 to nonatomic invariant probability measures for $\mathcal{F C}_{B}$ and $\mathcal{F C}_{A}$. Conversely any nonatomic measure on those spaces gives zero mass to $\mathcal{N}_{B}, \mathcal{N}_{A}$ and so 
transports to an invariant probability measure for $\mathcal{F C}_{C}$. Minimality for $\mathcal{F C}_{C}$ implies essential minimality for $\mathcal{F C}_{B}, \mathcal{F C}_{A}$ since $\mathcal{N}_{B}, \mathcal{N}_{A}$ are countable sets.

(ii) Given one of the adic transformations $\left(\Sigma_{M}^{+} \backslash \mathcal{N}_{M, \mathcal{O}}, T_{M, \mathcal{O}}\right)$, we know from $(i)$ that $\mathcal{F} \mathcal{C}_{M}$ is essentially minimal and essentially uniquely ergodic. A nonatomic invariant measure for $\mathcal{F C}_{M}$ is invariant for $T_{M, \mathcal{O}}$, showing existence, while any invariant probability measure $\mu$ for $T_{M, \mathcal{O}}$ on $\Sigma_{M}^{+} \backslash \mathcal{N}_{M, \mathcal{O}}$ is invariant for $\mathcal{F C}_{M}$ (one checks this on cylinder sets, see Proposition 2.9 of [Fis08]), showing uniqueness. Hence $\left(\Sigma_{M}^{+} \backslash \mathcal{N}_{M, \mathcal{O}}, T_{M, \mathcal{O}}\right)$ is uniquely ergodic.

The orbits of $\mathcal{F C}_{A}$ are identical to the stable equivalence classes; we know these are all dense except for the equivalence class of $(. \overline{1})$ which is a fixed point for $\mathcal{F C}_{A}$. The same holds for $\mathcal{F C}_{B}$. Thus both these actions are essentially minimal.

So in particular the Chacon adic transformation $\left(\Sigma_{A}^{+} \backslash \mathcal{N}_{A}, T_{A}\right)$ is both minimal and uniquely ergodic.

Remark 5.2. The substitution defining the "final adic" above comes from [Fer02] where the Chacon substitution dynamical system was compared to that substitution dynamical system. Our proof here is essentially an "adic version" of that idea.

A point that we find interesting in the above construction is that though the map $\Phi$ conjugates the adic transformations, it does not congugate the shift maps. We note that this is a one-sided phenomenon, as the corresponding block code on the two-sided spaces does conjugate the shifts as well. A geometrical view of this will be given in a later paper.

We mention that a quick (but not self-contained) proof of the minimality and unique ergodicity of the Chacon adic transformation can be given which is based on the following three facts: Mosse's recognizability theorem ([Mos92], [Mos96], [Hos00]), the canonical correspondence between stationary adic transformations and substitution dynamical systems for the primitive, recognizable case, first noted by Livshits, [Liv87], [Liv88], and the known fact (proved in any of several known ways [Fer02]) that the Chacon substitution dynamical system is minimal and uniquely ergodic. In brief, there is always a canonical factor map from the edge path space $\Sigma_{A}^{+} \backslash \mathcal{N}$ to the biinfinite substitution dynamical system $(\Omega, S)$ with left shift map $S$; this is bijective iff the substitution is recognizable, which is implied by aperiodicity via Mosse's theorem. In contrast to our purely adic proof, the validity of this approach depends on the order $\mathcal{O}$, as recognizability is not independent of the particular substitution chosen. For an example of this, the Thue-Morse substitution $\rho(0)=01, \rho(1)=10$ and the substitution $\widetilde{\rho}(0)=01, \widetilde{\rho}(1)=01$ have the same matrix $\left[\begin{array}{ll}1 & 1 \\ 1 & 1\end{array}\right]$, yet while the first is recognizable the second is not; indeed as an adic transformation $\widetilde{\rho}$ gives the adding machine, but the corresponding substitution dynamical system consists of a factor of this, a space with two points which are interchanged by the map.

\section{REFERENCES}

[AF01] Pierre Arnoux and Albert M. Fisher. The scenery flow for geometric structures on the torus: the linear setting. Chinese Ann. of Math., 4:427-470, 2001. 
$0 \multimap b \rightarrow 0 \multimap a \rightarrow 0 \multimap a \rightarrow 0 \multimap a \rightarrow 0 \multimap d \rightarrow 0-d \rightarrow 0 \multimap b \rightarrow 0$
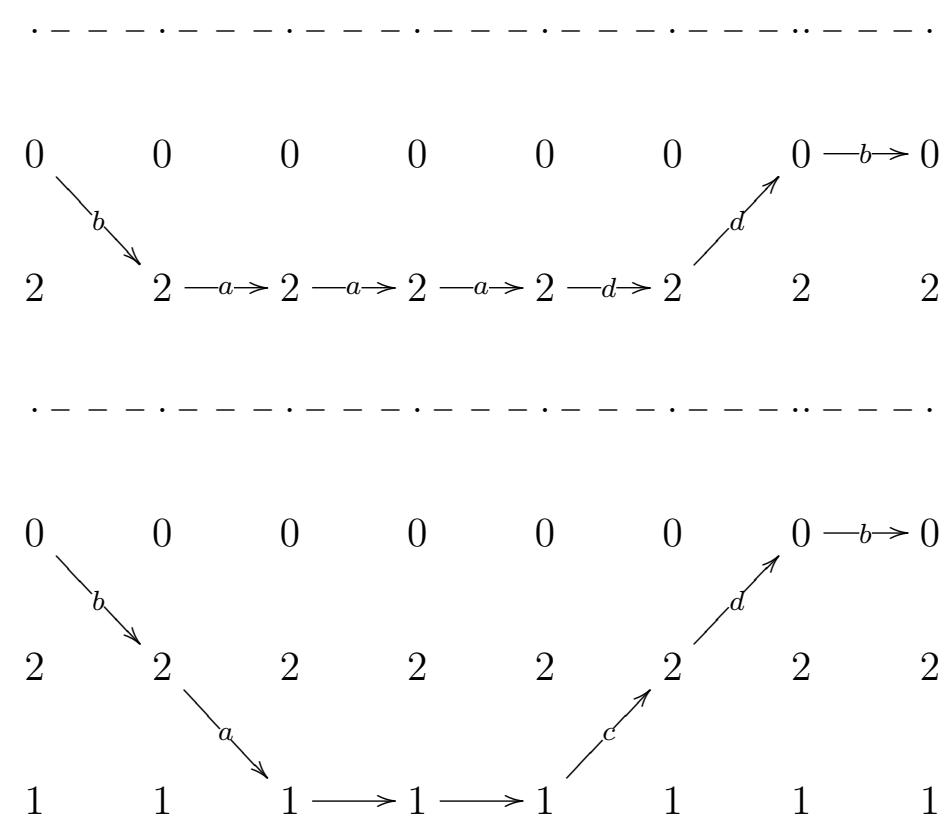

Figure 6 . How a path maps from $\Sigma_{A}^{+}$to $\Sigma_{B}^{+}$to $\Sigma_{C}^{+}$.

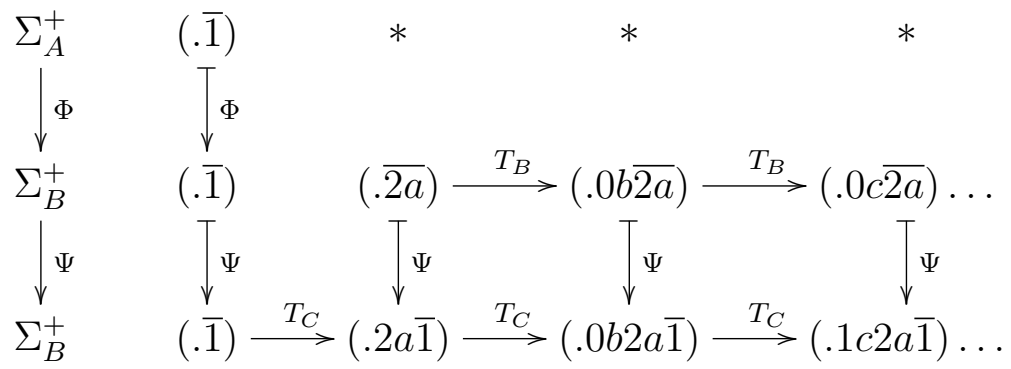

Figure 7 . How the exceptional edge path $(.111 \ldots)$ is mapped; $*$ indicates there is no preimage for the map $\Phi$.

[AF05] Pierre Arnoux and Albert M. Fisher. Anosov families, renormalization and nonstationary subshifts. Erg. Th. and Dyn. Sys., 25:661-709, 2005.

[BKMS09] S. Bezuglyi, J. Kwiatkowski, K. Medynets, and B. Solomyak. Invariant measures on stationary Bratteli diagrams. Ergodic Theory and Dynamical Sys., 2009. to appear.

[BM77] Rufus Bowen and Brian Marcus. Unique ergodicity for horocycle foliations. Israel Jour. Math., 26(1):43-67, 1977.

[Cha69] R.V. Chacon. Weakly mixing transformations which are not strongly mixing. Proc. Amer. Math. Soc., 22:559-562, 1969.

[Fer95] Sebastien Ferenczi. Les transformations de Chacon:combinatiore, structure géoétrique, lien aves les systèmes de complexité $2 n+1$. Bull. SMF, 123(2):272-292, 1995.

[Fer97] Sebastien Ferenczi. Systems of finite rank. Colloq. Math., 73(1):35-65, 1997. 


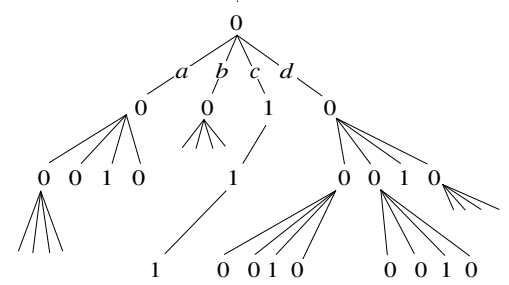

Figure 8. A stable tree for the Chacon adic; $0^{\text {th }}$ coordinate is lowest; the adic transformation maps paths from left to right following these lowest vertices.

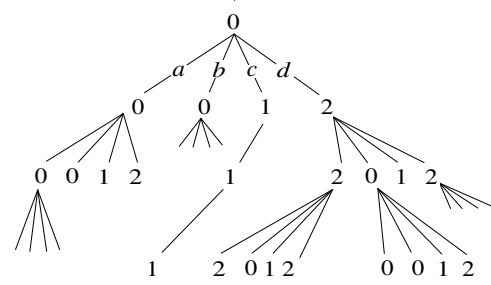

FiguRE 9. The corresponding stable tree for the intermediate adic.

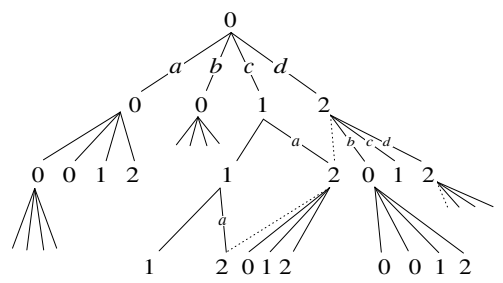

FiguRE 10. The corresponding stable tree for the final adic, showing branches erased and added by the map $\Psi$.

[Fer02] Sebastien Ferenczi. Substitutions and symbolic dynamical systems. In A. Siegel V. Berthe, P. Arnoux, editor, Substitutions in Dynamics, Arithmetics and Combinatorics, number 1794 in Lecture Notes in Math. Springer, 2002.

[Fis] Albert M. Fisher. Abelian differentials, interval exchanges, and adic transformations. in preparation, 2008.

[Fis92] Albert M. Fisher. Integer Cantor sets and an order-two ergodic theorem. Ergod. Th. and Dynam. Sys., 13:45-64, 1992.

[Fis08] Albert M. Fisher. Nonstationary mixing and the unique ergodicity of adic transformations, 2008. preprint, submitted.

[Fur61] Hillel Furstenberg. Strict ergodicity and transformation of the torus. Amer Jour Math, 83:573-601, 1961.

[Fur73] Hillel Furstenberg. The unique ergodicity of the horocycle flow. In A. Beck, editor, Recent Advances in Topological Dynamics, volume 318 of Springer Lecture Notes, pages 95-115. Springer Verlag, 1973. 
[FZ06] S. Ferenczi and L. Zamboni. Examples of 4-interval exchange transformations, 2006. Preliminary version.

[Hos00] Bernard Host. Substitution subshifts and Bratteli diagrams. In A. Maas F. Blanchard and A. Nogueira, editors, Topics in Symbolic Dynamics and Applications, number 279 in London Math. Soc. Lecture Note Series. Cambridge Univ. Press, 2000.

[Jew70] R.I. Jewett. The prevalence of uniquely ergodic systems. J. Math. Mech., 19:717-729, 1970.

[Kea75] Michael Keane. Interval exchange transformations. Math. Zeitschrift, 141(1):25-31, 1975.

[Kea77] Michael Keane. Non-ergodic interval exchange transformations. Israel Jour. Math., 26(2):188-196, 1977.

[KN76] Harvey B. Keynes and Dan Newton. A "minimal", non-uniquely ergodic interval exchange transformation. Math. Zeitschr., 148(2):101-105, June 1976.

[Kri70] W. Krieger. On unique ergodicity. Proc. Sixth Berkeley Symposium on Math. Stat. and Prob., pages 327-346, 1970.

[Liv87] A. N. Livshits. On the spectra of adic transformations of Markov compacta. Russian Math. Survey, 42(3):222-223, 1987.

[Liv88] A. N. Livshits. A sufficient condition for weak mixing of substitutions and stationary adic transformations. Math Notes, 44:920-925, 1988.

[LM95] Douglas Lind and Brian Marcus. Symbolic dynamics and coding. Cambridge University Press, 1995.

[LV85] A.A. Lodkin and A.M. Vershik. Approximation for actions of amenable groups and transversal automrophisms, lecture notes in math., vol. 1132, springer- verlag, new york, 1985, pp. 331346. In H. Araki, C.C. Moore, S. Stratila, and D. Voiculescu, editors, Operator Algebras and their connections with Topology and Ergodic Theory, volume 1132 of Lecture Noted in Math., pages 331-346. Springer Verlag, 1985.

[LV92] A. N. Livshits and A. M. Vershik. Adic models of ergodic transformations, spectral theory, substitutions, and related topics. Advances in Soviet Mathematics, 9:185-204, 1992. Representation theory and Dynamical Systems.

[Mas82] Howard Masur. Interval exchange transformations and measured foliations. Annals of Math., 115:169-200, 1982.

[Mos92] Brigitte Mossé. Puissances de mots et reconnaissabilité des points fixes d'une substitution. Theoret. Comput. Sci., 99(2):327-334, 1992.

[Mos96] Brigitte Mossé. Reconnaissabilité des substitutions et complexité des suites automatiques. Bull. Soc. Math. France, 124(2):329-346, 1996.

[MP05] Xavier Mela and Karl Petersen. Dynamical properties of the Pascal adic transformation. Ergodic Theory Dynam. Systems, 25(1):227-256, 2005.

[Vee78] William A. Veech. Interval exchange transformations. Journal d'Anayse of Mathé matique, 33:222-272, 1978.

[Vee82] William A. Veech. Gauss measures for transformations on the space of interval exchange maps. Annals of Mathematics, 115:201-242, 1982.

[Ver81] Anatoly M. Vershik. Uniform algebraic approximation of shift and multiplication operators. Soviet Math. Dokl., 24(1):101-103, 1981.

[Ver89] A. M. Vershik. A new model of the ergodic transformations. In Dynamical Sustems and Ergodic Theory, volume 23 of Banach Center Publications, pages 381-384, Warsaw, 1989. PWN- Polish Scientific Publishers.

[Ver94] Anatoly M. Vershik. Locally transversal symbolic dynamics. Algebra i Analiz, 6:94-106, 1994. in russian.

[Ver95a] Anatoly M. Vershik. The adic realizations of the ergodic actions with the homeomorphisms of the Markov compact and the ordered Bratteli diagrams. Teor. Predstav. Din. Systemy. Kombin. i Algoritm. Metody., 1:120-126, 1995.

[Ver95b] Anatoly M. Vershik. Locally transversal symbolic dynamics. St. Petersburg Math Journal, 6:529-540, 1995. translation of 1994 Russian version. 
[Via06] Marcelo Viana. Dynamics of interval exchange transformations and Teichmuller flows. lecture notes, August 2006. http://w3.impa.br/ viana/out/ietf.pdf.

[Wal82] P. Walters. An Introduction to Ergodic Theory. Springer Verlag, New York/Berlin, 1982.

Sebastien Ferenczi Institut de Mathématiques de Luminy (UPR 9016), 163 Avenue de Luminy, case 93013288 Marseille Cedex 9, France

E-mail address: ferenczi(lowerbar)sebastien@yahoo.fr

Albert M. Fisher, Dept Mat ime-UsP, Caixa Postal 66281, CeP 05315-970 São Paulo, Brazil

$U R L$ : http://ime.usp.br/ afisher

E-mail address: fisher(lowerbar)alby@yahoo.fr

Marina Talet C.M.I. Université de Provence LATP, CNRS-UMR 6632 F-13453 MarSeille Cedex 13 France

E-mail address: marina@cmi.univ-mrs.fr 